\title{
Effects of the traditional Chinese medicine baicalein on the viability of random pattern skin flaps in rats
}

This article was published in the following Dove Press journal: Drug Design, Development and Therapy

\author{
Renjin $\operatorname{Lin}^{1,2, *}$ \\ Jinti $\operatorname{Lin}^{1,2, *}$ \\ Shihen $\mathrm{Li}^{1,2}$ \\ Jian Ding ${ }^{1,2}$ \\ Hongqiang $\mathrm{Wu}^{1,2}$ \\ Guangheng Xiang ${ }^{1,2}$ \\ Shi Li $i^{1,2}$ \\ Yijia Huang ${ }^{2}$ \\ Dingsheng $\operatorname{Lin}^{1,2}$ \\ Weiyang Gao',2 \\ Jianzhong Kong ${ }^{1,2}$ \\ Huazi Xu ${ }^{1,2}$ \\ Kailiang Zhou ${ }^{1,2}$ \\ 'Department of Orthopaedics, \\ The Second Affiliated Hospital \\ and Yuying Children's Hospital \\ of Wenzhou Medical University, \\ Wenzhou 325027, China; ${ }^{2}$ The Second \\ Clinical Medical College of Wenzhou \\ Medical University, Wenzhou 325027, \\ China \\ *These authors contributed equally \\ to this work
}

Correspondence: Kailiang Zhou Department of Orthopaedics, The Second Affiliated Hospital and Yuying Children's Hospital of Wenzhou Medical University, 109 West Xueyuan Road,

Wenzhou 325000, Zhejiang, China

Tel +8 657788002815

Email zhoukailiang@wmu.edu.cn
Background: Random skin flaps are routinely placed during plastic and reconstructive surgery, but the distal areas often develop ischemia and necrosis. Baicalein, a major flavonoid extracted from the traditional Chinese herbal medicine huangqin, Scutellaria baicalensis Georgi, may improve flap viability.

Materials and methods: Rats were randomly divided into baicalein and control groups and they underwent placement of modified McFarlane flaps after intraperitoneal administration of baicalein or vehicle. Flap survival and water content were measured 7 days later, as were angiogenesis, apoptosis, and oxidative stress in ischemic flaps.

Results: Baicalein promoted flap survival, reduced edema, increased mean vessel density, and enhanced vascular endothelial growth factor production at both the translational and transcriptional levels. Baicalein reduced caspase 3 cleavage, increased superoxidase dismutase and glutathione levels, and decreased the malondialdehyde level.

Conclusion: Baicalein promoted flap viability by stimulating angiogenesis and inhibiting apoptosis and oxidation.

Keywords: baicalein, random skin flap, angiogenesis, apoptosis, oxidation

\section{Introduction}

Random skin flaps are routinely placed during plastic and reconstructive surgery to restore superficial skin defects. ${ }^{1}$ Despite advances in surgical techniques, the length-towidth ratio cannot exceed $2: 1$; otherwise, the distal areas become necrotic. ${ }^{2}$ The blood supply gradually decreases distally, triggering necrosis. ${ }^{3,4}$ After vascular regeneration, flap reperfusion and restoration of the blood supply trigger ischemia/reperfusion injury, also promoting necrosis caused by oxidative stress and apoptosis. ${ }^{5-7}$ Agents promoting angiogenesis and reducing oxidative stress and apoptosis would improve flap viability.

Baicalein (5,6,7-trihydroxy-2-phenyl-4H-1-benzopyran-4-one) is a major flavonoid of the traditional Chinese herbal medicine huangqin, Scutellaria baicalensis Georgi. ${ }^{8}$ A similar flavonoid, casticin (3,5-dihydroxy-3,4,6,7-tetramethoxyflavone), promotes angiogenesis. ${ }^{9,10}$ Baicalein stimulates angiogenesis by affecting $\mathrm{Wnt} / \beta$-catenin signaling, inhibits oxidative stress by enhancing the activities of antioxidant enzymes including superoxide dismutase (SOD) and glutathione (GSH) peroxidase, and inhibits apoptosis by regulating the phosphoinositide-3-kinase/Akt (PI3K/Akt) pathway. ${ }^{11-13}$ Baicalein is widely used to treat ischemic diseases such as myocardial ischemia and ischemic stroke. ${ }^{14,15}$ Because random pattern skin flaps are prone to ischemia and necrosis, we hypothesized that baicalein can improve flap survival. 
In this study, we used an in vivo assay to explore whether baicalein promotes flap viability in rats and to identify the pharmacological targets. Our work provides new insight into the therapeutic utility of baicalein, which inhibited flap ischemia and necrosis.

\section{Materials and methods Ethics statement}

All surgical procedures, treatments, and postoperative care were approved by the Wenzhou Medical University Ethics Committee and conformed strictly to the International Health and Medical Research Guidelines for Animal Welfare.

\section{Drugs and reagents}

Baicalein $\left(\mathrm{C}_{15} \mathrm{H}_{10} \mathrm{O}_{5}\right.$, HPLC purity $\left.>98 \%\right)$ was purchased from Tauto Biotechnology (Shanghai, China). H\&E staining kit, pentobarbital sodium, PBS, and diaminobenzidine were purchased from Solarbio Science \& Technology (Beijing, China). Primary antibodies against CD34 and vascular endothelial growth factor (VEGF) were obtained from Beijing Bioss Biotechnology (Beijing, China). SOD, GSH, and malondialdehyde (MDA) assay kits were purchased from the Nanjing Jiancheng Bioengineering Institute (Jiangsu, China). Primary antibodies against cleaved caspase 3 (CASP3) and $\beta$-actin were from Cell Signaling Technology (Beverly, MA, USA). A VEGF mRNA in situ hybridization kit was purchased from Boster Biological Technology (Wuhan, China). Horseradish peroxidase (HRP)-conjugated goat-anti-rabbit IgG was obtained from Boyun Biotechnology (Nanjing, China). A bicinchoninic acid test kit was purchased from Beyotime Biotechnology (Jiangsu, China). An electrochemiluminescence-plus immunodetection kit was obtained from Thermo Fisher Scientific (Waltham, MA, USA).

\section{Animals and drugs}

Sixty male Sprague Dawley rats were purchased from Wenzhou Medical University (license no SCXK [ZJ] 2005-0019). Before any experimental procedure, all rats were housed under a 12-hour light (06:00-18:00 hours) and 12-hour dark cycle (18:00-06:00 hours) with free access to regular food and water for 1 week. Then, the rats were randomly divided into two groups, the baicalein group $(n=30)$ and the control group ( $n=30)$. Baicalein was dissolved in dimethyl sulfoxide (DMSO) and immediately diluted with normal saline prior to injection (final concentration $10 \mathrm{mg} / \mathrm{mL}$; DMSO 10\% [v/v]). Before the surgical procedure, each rat in the test group received baicalein (100 $\mathrm{mg} / \mathrm{kg} /$ day intraperitoneally) for seven consecutive days; control rats received the same volume of DMSO-saline solution. After establishment of the modified McFarlane flap model, all rats were maintained on the same treatments until euthanasia.

\section{The modified McFarlane flap model}

After anesthesia with $2 \%(\mathrm{w} / \mathrm{v})$ pentobarbital sodium (40 mg/kg intraperitoneally), caudal-based $3 \times 9 \mathrm{~cm}$ areas were outlined on the back and the skin/panniculus carnosus was separated from the underlying fascia. Both sacral arteries were completely sectioned, and each flap was sutured back in the original position using 4-0 silk. Each flap was divided into three equal zones: proximal (Area I), intermediate (Area II), and caudal (Area III) zones.

\section{Flap survival and edema assessment}

Macroscopic flap changes were observed daily for seven consecutive days. Appearance, color, texture, and hair condition were noted, and the surviving areas were quantified by superimposing photographs on a graph paper. Percentage of survival was calculated as:

$$
\frac{\text { Surviving area }}{\text { Total area }} \times 100 \% \text {. }
$$

Tissue edema was evaluated by measuring the water content. On Day 7 after surgery, the flaps were weighed, dried in an autoclave at $50^{\circ} \mathrm{C}$, and weighed again until no change was evident for 2 days. The percentage water content was calculated as:

$$
\frac{\text { Wet weight }- \text { Dry weight }}{\text { Wet weight }} \times 100 \% \text {. }
$$

\section{H\&E staining}

After sacrifice, tissue samples $(0.5 \times 0.5 \mathrm{~cm})$ were collected from the central parts of Area II flaps $(n=6)$, post-fixed in $4 \%(\mathrm{v} / \mathrm{v})$ paraformaldehyde for 24 hours, embedded in paraffin wax, and transversely sectioned. The sections (10 $\mu \mathrm{m}$ thick) were mounted on poly-L-lysine-coated slides for $\mathrm{H} \& \mathrm{E}$ staining as described previously. ${ }^{5}$ Under a light microscope (Olympus Corporation, Tokyo, Japan), tissue edema, leukocyte infiltration, and microvessels were scored. The number of microvessels per unit area $\left(/ \mathrm{mm}^{2}\right)$ was calculated as the mean vascular density (MVD). Six random fields from three random sections from each sample were evaluated.

\section{Immunohistochemistry}

Sections from the central parts of Area II flaps $(n=6)$ were deparaffinized in xylene, rehydrated in graded ethanol baths, and subjected to immunohistochemical analyses. After washing, the sections were covered with $3 \%(\mathrm{v} / \mathrm{v}) \mathrm{H}_{2} \mathrm{O}_{2}$ to block 
endogenous peroxidase, placed in $10.2 \mathrm{mM}$ sodium citrate buffer at $95^{\circ} \mathrm{C}$ for 30 minutes, blocked with $5 \%(\mathrm{w} / \mathrm{v})$ bovine serum albumin for 15 minutes, incubated with primary antibodies against CD34 (1:200), VEGF (1:200), and SOD (1:100) at $4^{\circ} \mathrm{C}$ overnight, incubated with HRP-conjugated IgG secondary antibody $(1: 1,000)$, stained with 3,3'-diaminobenzidine, counterstained with hematoxylin, and sealed. Under a light microscope, six random fields from three random sections from each flap were examined. Absorbances were quantified using Image J software (NIH, Bethesda, MD, USA) to estimate VEGF and SOD expression. The number of CD34-positive blood vessels per unit area $\left(\mathrm{mm}^{2}\right)$ was calculated.

\section{In situ hybridization}

An in situ hybridization kit was used to detect VEGF mRNA in the flap tissue. The probes for VEGF mRNA were $5^{\prime}$-GCTCT ACCTC CACCA TGCCA AGTGG TCCCA-3', 5'-GACCC TGGTG GACAT CTTCC AGGAG TACCC-3', and 5'-GCAGC TTGAG TTAAA CGAAC GTACT TGCAG-3'. In situ hybridization was performed as described previously. ${ }^{16}$ After staining with 3,3'-diaminobenzidine, the sections were counterstained with hematoxylin and sealed. Under a light microscope, six random fields from three random sections from each flap were examined. The absorbance, indicating the level of VEGF transcription, was quantified using ImageJ software.

\section{Western blotting}

On Day 7 after surgery, samples $(0.5 \times 0.5 \mathrm{~cm})$ were separated from the central regions of Area II flaps $(n=6)$ and stored at $-80^{\circ} \mathrm{C}$ prior to Western blotting. Proteins were extracted from the flap homogenates and quantitated using a bicinchoninic acid kit. Proteins $(60 \mu \mathrm{g})$ were separated on $12 \%(\mathrm{w} / \mathrm{v})$ gels and transferred to polyvinylidene fluoride membranes (Roche Applied Science, Indianapolis, IN, USA). The membranes were blocked with $10 \%(\mathrm{w} / \mathrm{v})$ nonfat milk and incubated with primary antibodies (anti-VEGF [1:1,000], anti-cleaved CASP3 [1:500], anti-SOD [1:500], and anti- $\beta$-actin $[1: 1,000])$ overnight at $4{ }^{\circ} \mathrm{C}$, then incubated with the HRP-conjugated IgG secondary antibody $(1: 5,000)$ at room temperature for 1.5 hours, and visualized using the electrochemiluminescence-Plus immunodetection kit. The protein band intensities were quantified using Image Lab ver. 3.0 software (Bio-Rad, Hercules, CA, USA).

\section{SOD activity, GSH level, and MDA content}

On Day 7 after surgery, tissue samples $(0.5 \times 0.5 \mathrm{~cm})$ were obtained from the central regions of Area II $(n=6)$ and assayed for SOD activity, GSH level, and MDA content using commercial kits as described previously. ${ }^{17} \mathrm{SOD}, \mathrm{GSH}$, and
MDA were evaluated using the xanthine oxidase method, modified dithiobisnitrobenzoic acid test, and modified thiobarbituric acid test, respectively.

\section{Statistical analysis}

Statistical analyses were performed using SPSS ver. 19 software (IBM Corporation, Armonk, NY, USA). All data are expressed as mean $\pm \mathrm{SD}$. Mean values were compared between the groups using Student's $t$-test. Statistical significance was established at $* P<0.05$ and $* * P<0.01$.

\section{Consent for publication}

Hereby, we declare that our institutes are aware of the work and declare consent for publication of the manuscript. We, all the authors, have got the consent to publish the animal experimental data from the animal ethical committe of Wenzhou Medical University.

\section{Results \\ Baicalein improved flap survival and reduced tissue edema}

After surgery, all rats were observed for 7 days. On Day 3, Area III of each flap became purple and edematous, with black or brown nidi indicating distal ischemia; by day 7 , both Areas II and III were dark with scabbing and hardening, indicating necrosis (Figure 1A). The between-group difference in the surviving flap areas was significant $(P=0.01)$; the percentages of survival were $81.00 \% \pm 11.74 \%$ and $62.17 \% \pm 7.63 \%$ in the baicalein and control groups, respectively (Figure 1C). The flaps were detached and the inner sides examined. As shown in Figure 1B, Areas II and III of the control flaps were swollen and bruised, with serious subcutaneous venous blood stasis; these signs of edema were ameliorated in the baicalein group. The percentage of flap water content was significantly lower in the baicalein group $(48.17 \% \pm 7.63 \%)$ than in the control group $(61.00 \% \pm 10.20 \%$, $P=0.035$; Figure 1D). Thus, baicalein ameliorated edema.

\section{Baicalein promoted angiogenesis in ischemic flaps}

On Day 7 after surgery, Area II of each flap was stained with H\&E. As shown in Figure 2A, the baicalein group exhibited more neovascularization and subcutaneous hemorrhage and less necrosis compared with the control group. The MVD was significantly greater in the baicalein group $\left(35.29 \pm 12.16 / \mathrm{mm}^{2}\right)$ than in the control group $\left(21.37 \pm 7.05 / \mathrm{mm}^{2}, P=0.041\right.$; Figure 2C). Immunohistochemical analysis of CD34 was used to label vessel endothelial cells. The baicalein group had more CD34-positive vessels than did the control 
A
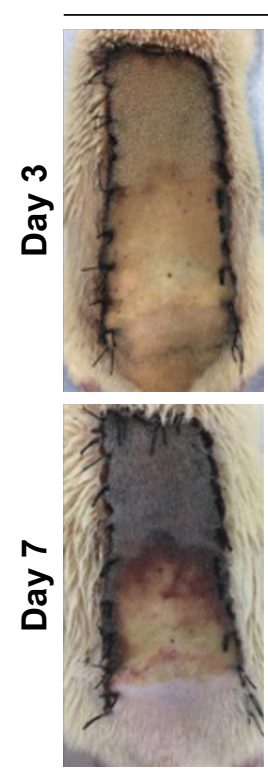

B

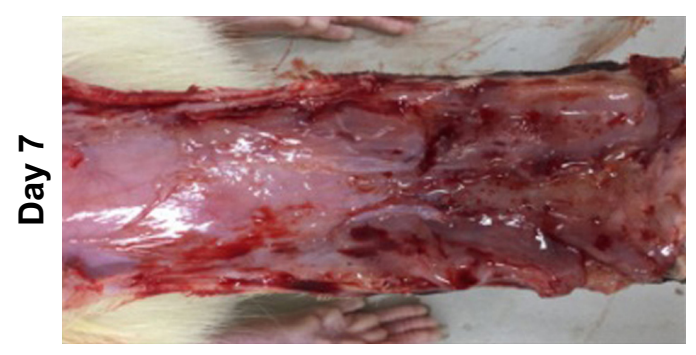

Control
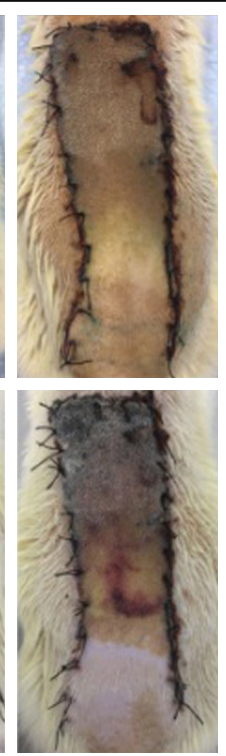

Control

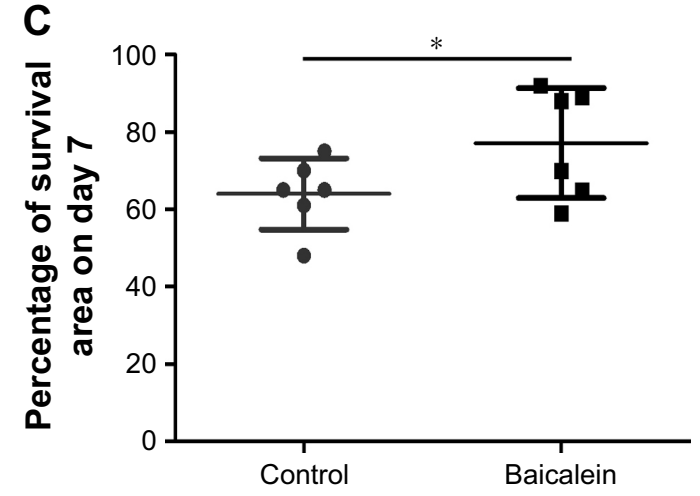

Baicalein
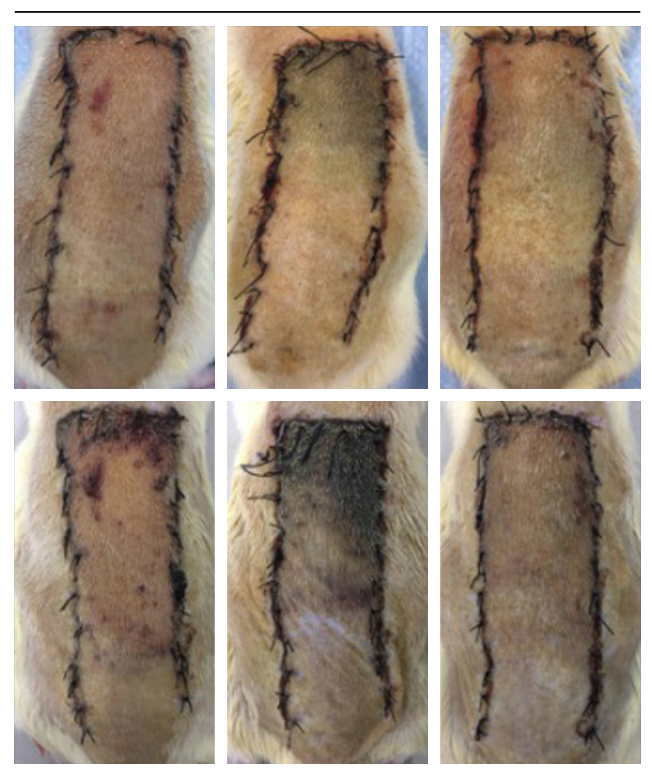

\section{Baicalein}

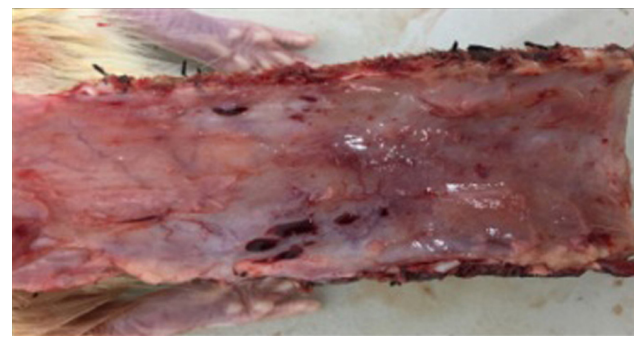

D

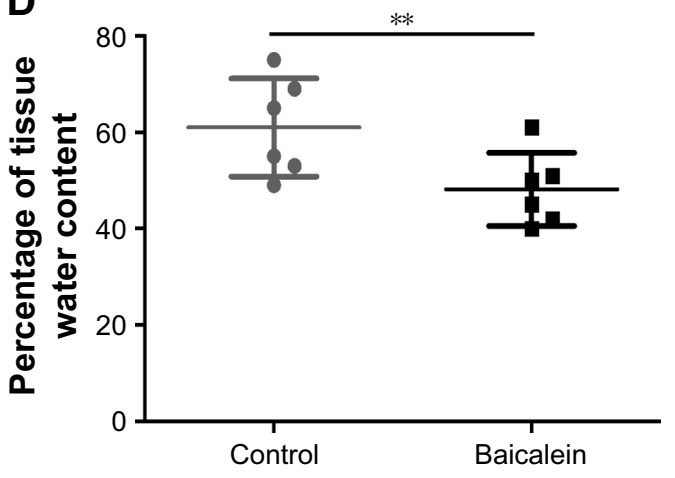

Figure I Baicalein improved flap survival and reduced edema.

Notes: (A) Digital photographs of the flaps taken on Days 3 and 7. (B) Digital photographs of the inner sides of the flaps taken on Day 7 to measure edema. (C) Survival percentages. (D) Tissue water content. Values are mean $\pm S D, n=6$ per group. Significant differences between the groups are indicated by $* P<0.05$ and $* * P<0.0$ I.

group (Figure 2B). Baicalein significantly increased the MVD to $32.86 \pm 8.23 / \mathrm{mm}^{2}$ compared with $18.84 \pm 4.94 / \mathrm{mm}^{2}$ in controls $(P=0.005$; Figure $2 \mathrm{D})$.

\section{Baicalein increased VEGF mRNA and protein levels in ischemic flap areas}

On Day 7 after surgery, in situ hybridization of VEGF mRNA in Area II of all flaps (Figure 3A) showed that more VEGF mRNA was synthesized by keratinocytes and fibroblasts in the vascular structures of the cutis and dermis in the baicalein group compared with the control group, as reflected by the higher OD in the former group ( $P=0.008$; Figure $3 \mathrm{~B})$. We also detected VEGF expression by immunochemistry. Greater VEGF expression in dermal vessels and stromal cells was apparent in the baicalein group, as indicated by the higher OD ( $P=0.028$; Figure $3 \mathrm{C}$ and $\mathrm{D})$. Western blotting showed higher VEGF protein levels in the baicalein group than in the control group ( $P=0.007$; Figure $3 \mathrm{E}$ and $\mathrm{F}$ ). 
A
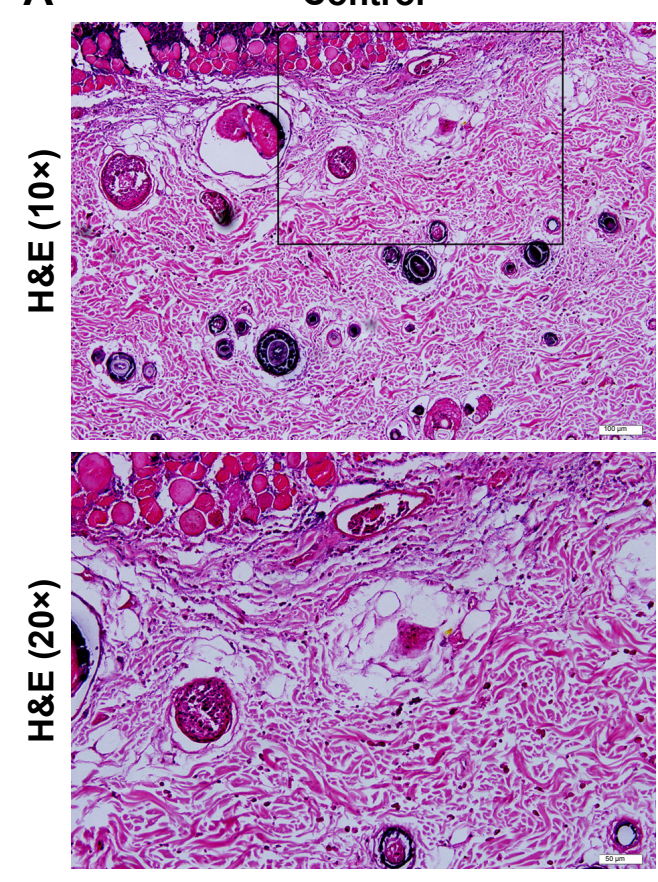

B
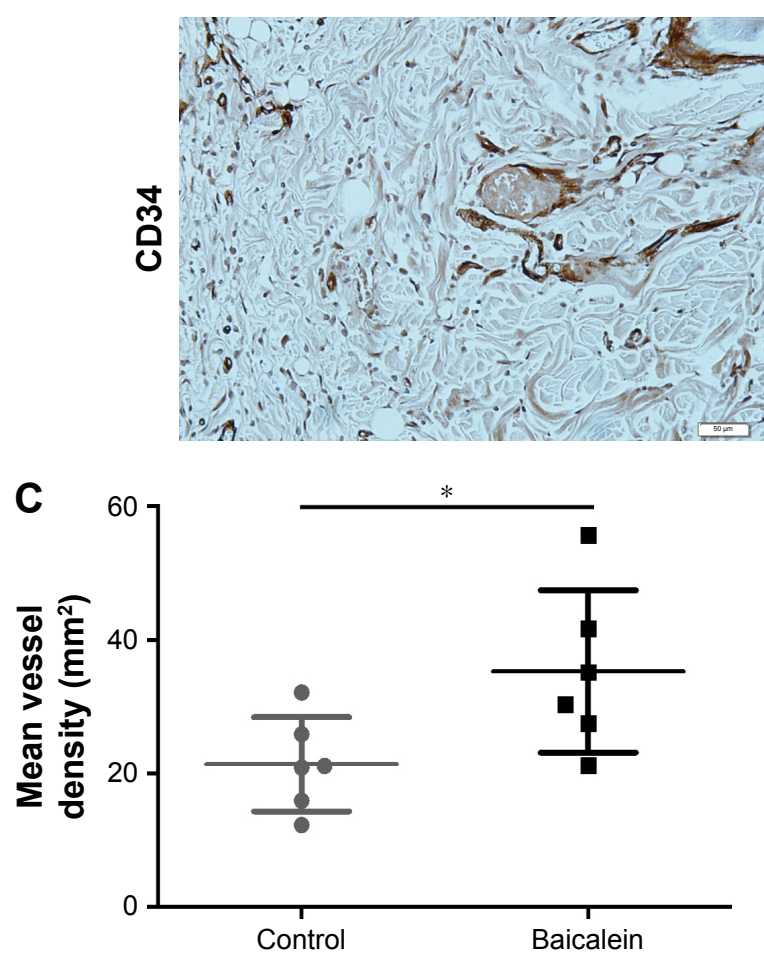

Baicalein
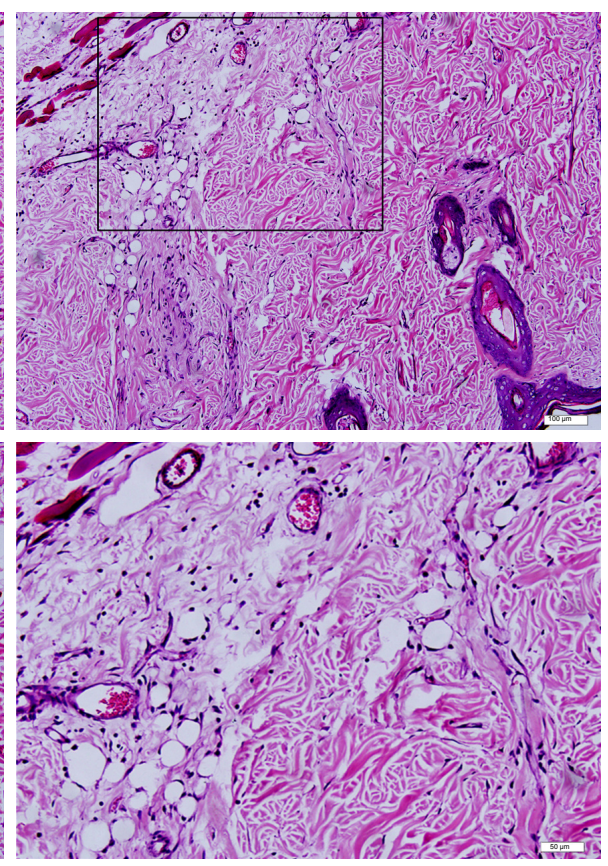

Baicalein
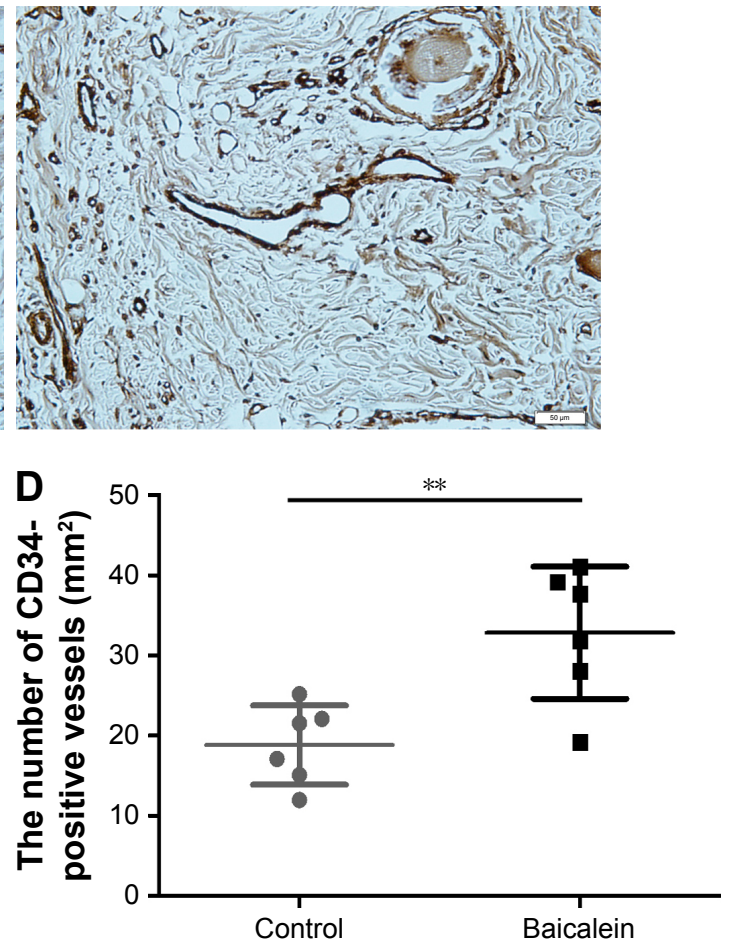

Figure 2 Baicalein promoted angiogenesis in flap ischemic areas.

Notes: (A) On Day 7, angiogenesis in the ischemic flap areas (Areas II) was evident by H\&E staining (original magnification, $\times 100$ and $\times 200$; scale bars, 50 and I00 $\mu \mathrm{m}$ ). (B) Immunochemical detection of CD34, which is a marker of angiogenesis (original magnification, $\times 200$; scale bar, $50 \mu \mathrm{m}$ ). (C) MVDs assessed by H\&E staining. (D) The numbers of CD34-positive vessels. Values are mean $\pm S D, n=6$ per group. Significant differences between the groups are indicated by $* P<0.05$ and $* * P<0.01$.

\section{Baicalein reduced apoptosis in ischemic flap areas}

On Day 7 after surgery, immunohistochemical analysis of cleaved CASP3 was performed on ischemic flap areas.
Cleaved CASP3 was detected in dermal fibroblasts and vascular structures and striated muscle cells of the myometrium, but less so in the baicalein group than in the control group, as reflected by the lower OD in the former $(P=0.022$; 

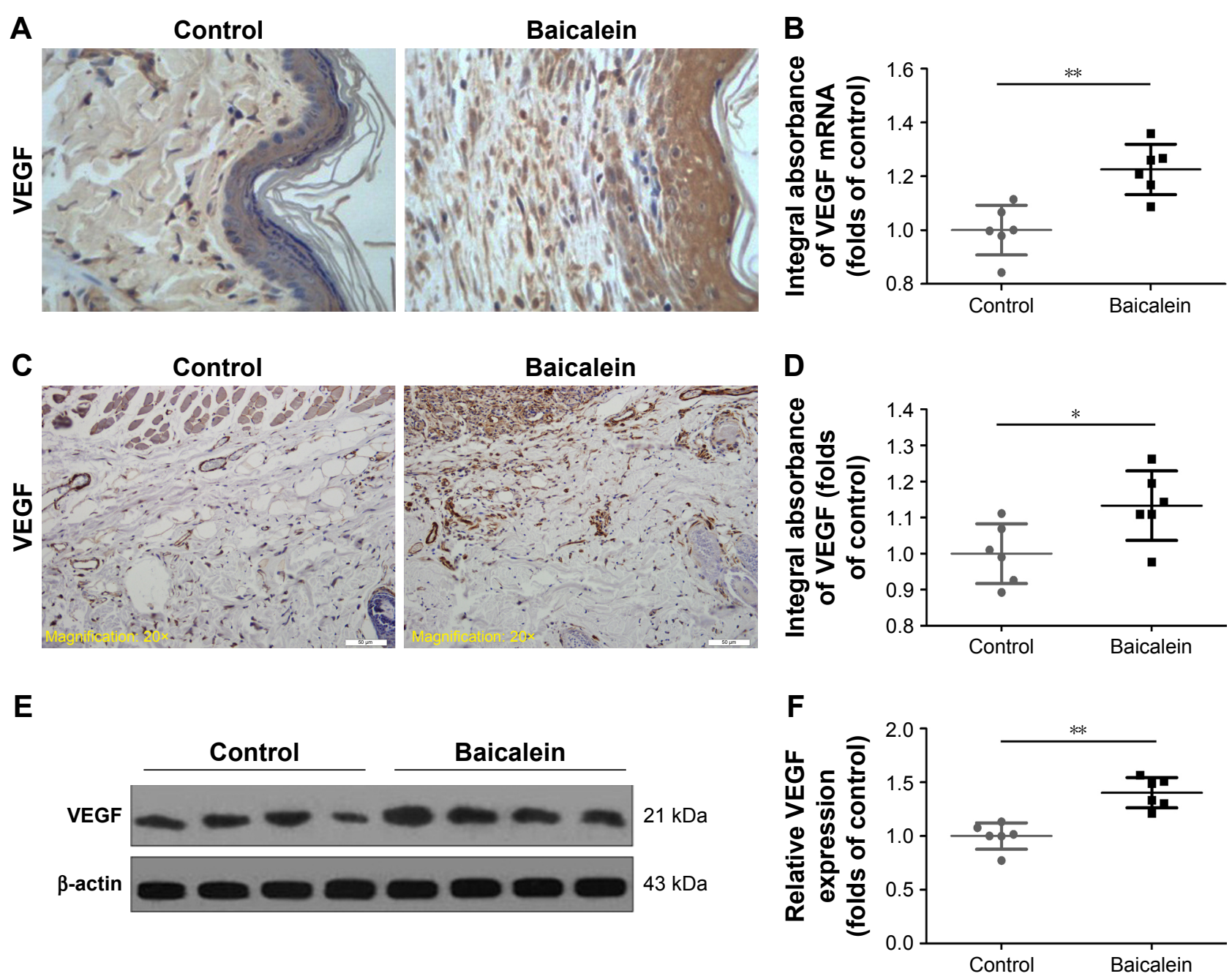

Figure 3 Baicalein increased VEGF mRNA/protein levels in ischemic flap regions.

Notes: (A) In situ hybridization of VEGF mRNA on Day 7 (original magnification, $\times 400$ ). (B) ODs indicative of VEGF transcription levels. (C) Immunohistochemical VEGF expression on Day 7 (original magnification, $\times 200$; scale bar, $50 \mu \mathrm{m}$ ). (D) ODs indicative of VEGF mRNA level. (E) Western blotting for VEGF on Day 7. Gels were run under the same experimental conditions, and cropped images of the blots are presented. (F) ODs indicative of VEGF protein expression. Values are expressed as mean $\pm S D$, $\mathrm{n}=6$ per group. Significant differences between the groups are indicated by $* \mathrm{P}<0.05$ and $* * P<0.01$.

Figure 4A and C). Western blotting for CASP3 protein revealed significantly lower levels in the baicalein group than in the control group $(P=0.01$; Figure $4 \mathrm{~B}$ and $\mathrm{D})$.

\section{Baicalein reduced oxidative stress in flap ischemic regions}

On Day 7 after surgery, immunohistochemical and Western blot analyses of SOD were performed to evaluate the oxidative stress status in Area II of each flap. Compared with the control group, the baicalein group expressed more SOD in vessels and stromal cells and striated muscle cells, as indicated by the higher OD in the baicalein group $(P=0.045$; Figure 5A and B). Western blotting showed higher SOD protein levels in the baicalein group than in the control group (Figures 5C and 3D; $P=0.006$ ). SOD, GSH, and MDA levels were also evaluated using assay kits. The SOD level in the baicalein group was $54.29 \pm 8.01 \mathrm{U} / \mathrm{mg}$ protein, higher than that in the control group $(40.88 \pm 8.66 \mathrm{U} / \mathrm{mg}$ protein, $P=0.019$; Figure 5E). Baicalein treatment was associated with a higher level of GSH $(2.72 \pm 0.40 \mathrm{nmol} / \mathrm{mg}$ protein) compared with the control level $(2.22 \pm 0.35 \mathrm{nmol} / \mathrm{mg}$ protein, $P=0.046$; Figure $5 \mathrm{~F}$ ). The MDA level was significantly lower in the baicalein group $(44.16 \pm 8.09 \mathrm{nmol} / \mathrm{mg}$ protein) than in the control group $(60.44 \pm 6.99 \mathrm{nmol} / \mathrm{mg}$ protein, $P=0.004$; Figure 5G).

\section{Discussion}

Baicalein, the principal active constituent of the roots of huangqin, S. baicalensis Georgi, exerts beneficial effects in patients with ischemic diseases such as liver ischemia/reperfusion injury, and retinal and cerebral ischemia. ${ }^{18-20}$ Baicalein exhibits proangiogenic, antioxidative, and antiapoptotic 
A

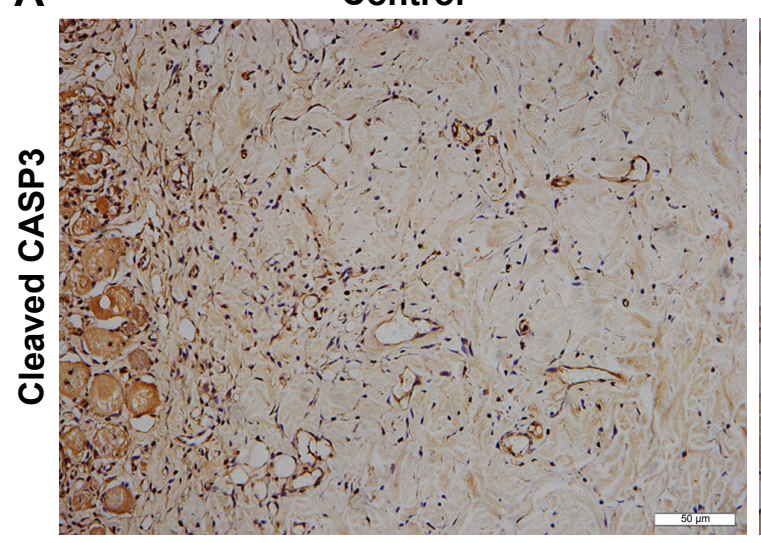

\section{Baicalein}

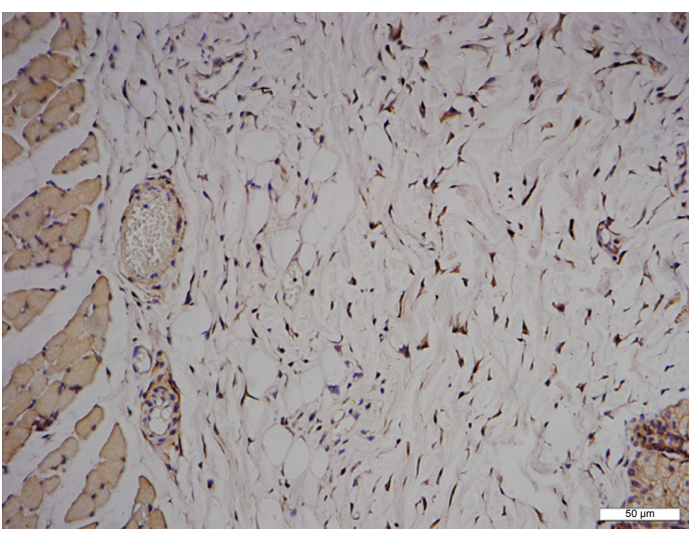

B

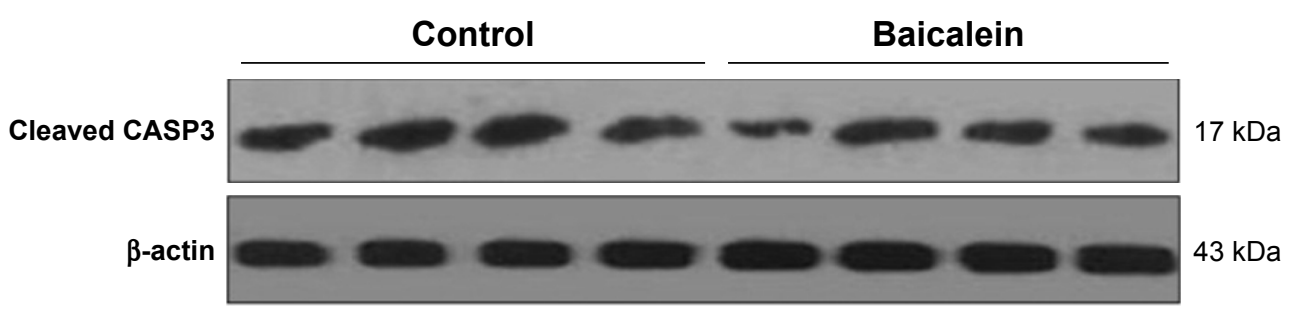

C

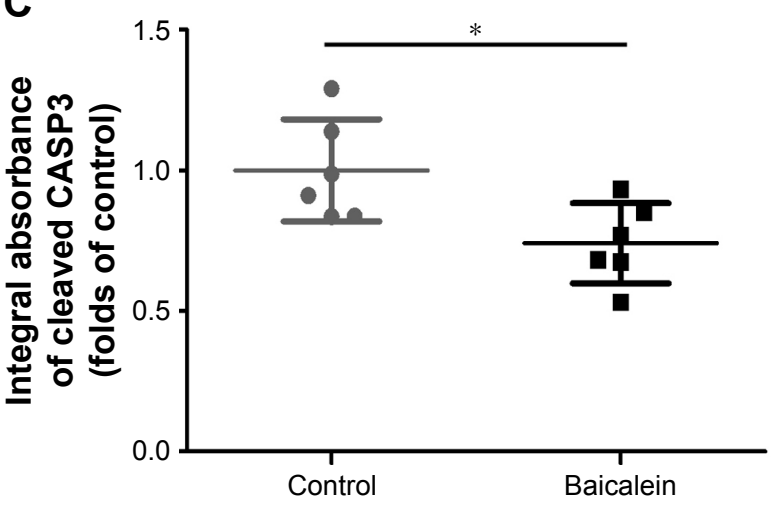

D

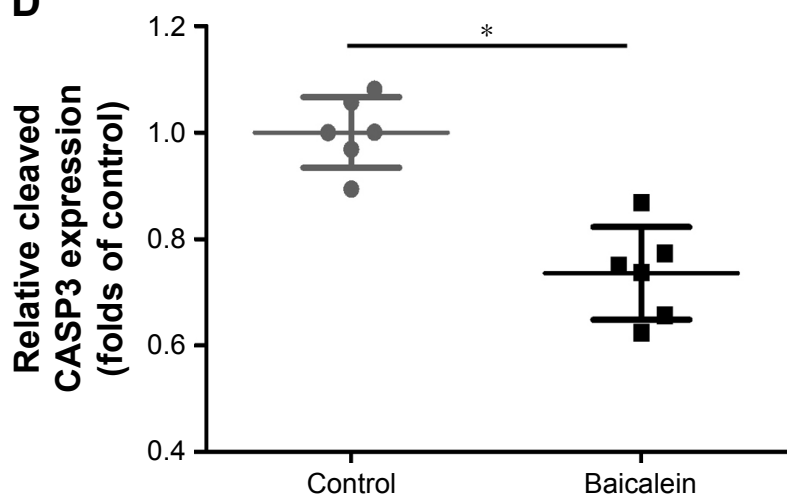

Figure 4 Baicalein reduced apoptosis in ischemic flap regions.

Notes: (A) Immunohistochemical evaluation of cleaved CASP3 levels on Day 7 (original magnification, $\times 200 ;$ scale bar, $50 \mu \mathrm{m}$ ). (B) Western blotting for cleaved CASP3 on Day 7. Gels were run under the same experimental conditions, and cropped blots are presented. (C) ODs indicative of VEGF protein expression. (D) ODs were quantified and analyzed. Values are expressed as mean $\pm S D ; n=6$ per group. Significant differences between the groups are indicated by $* P<0.05$.

activities. Distal flap ischemia and necrosis induced by lack of blood are common in clinical practice. Few studies have addressed the effects of baicalein in this context. We found that baicalein significantly promoted random skin flap survival, which was associated with enhanced angiogenesis and reduced oxidative stress and apoptosis.

Several reports found that baicalein was proangiogenic both in vitro and in vivo. ${ }^{11,21}$ However, baicalein exerted antiangiogenic effects in models of retinal ischemia and several kinds of cancers. ${ }^{19,22}$ Therefore, the effects of baicalein on angiogenesis remain controversial and may depend on the disease microenvironment. Any effect of baicalein on random skin flap angiogenesis remains unknown. Our MVD data derived from H\&E staining and CD34 measurements show that baicalein promoted neovascularization of random skin flaps, prolonging survival. We also assessed the VEGF status and found that baicalein upregulated VEGF mRNA and protein levels in vessels and stromal cells. Western blotting also showed that VEGF levels were higher in the baicalein group than in the control group; baicalein promoted flap survival by enhancing VEGF-induced angiogenesis.

As distal ischemia develops in random skin flaps, blood resupply-induced ischemia/reperfusion injury contributes to necrosis. ${ }^{23,24}$ The mode of injury is complex, involving formation of oxygen free radicals, platelet aggregation, 
A

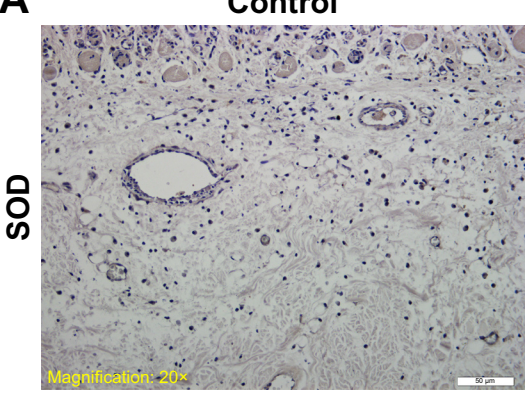

C
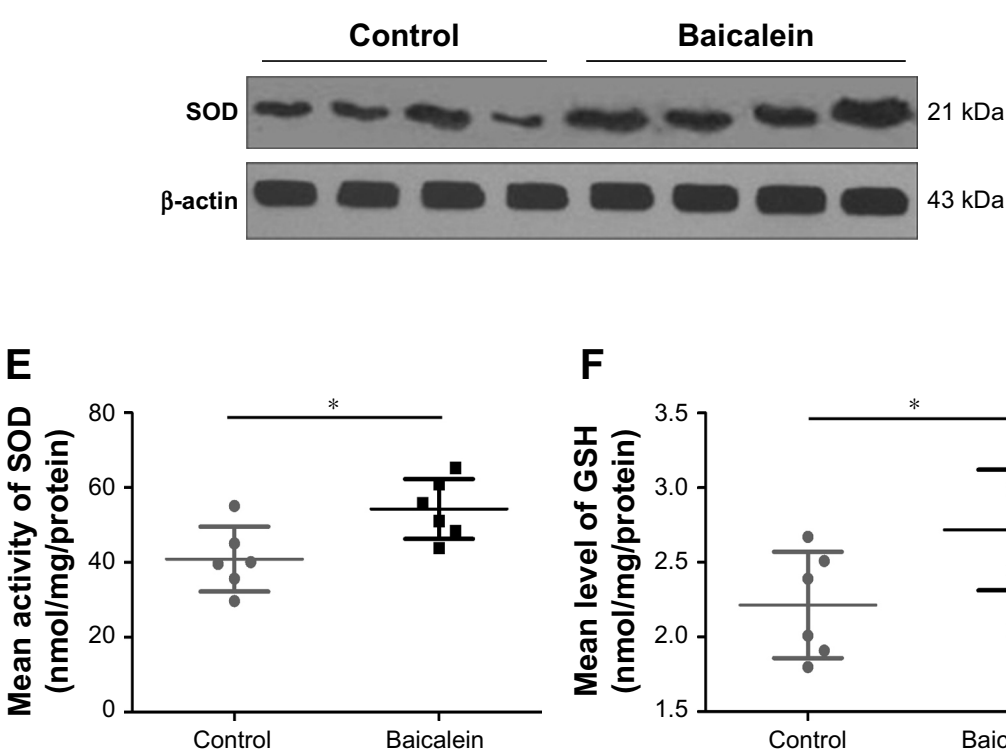

$\mathbf{F}$
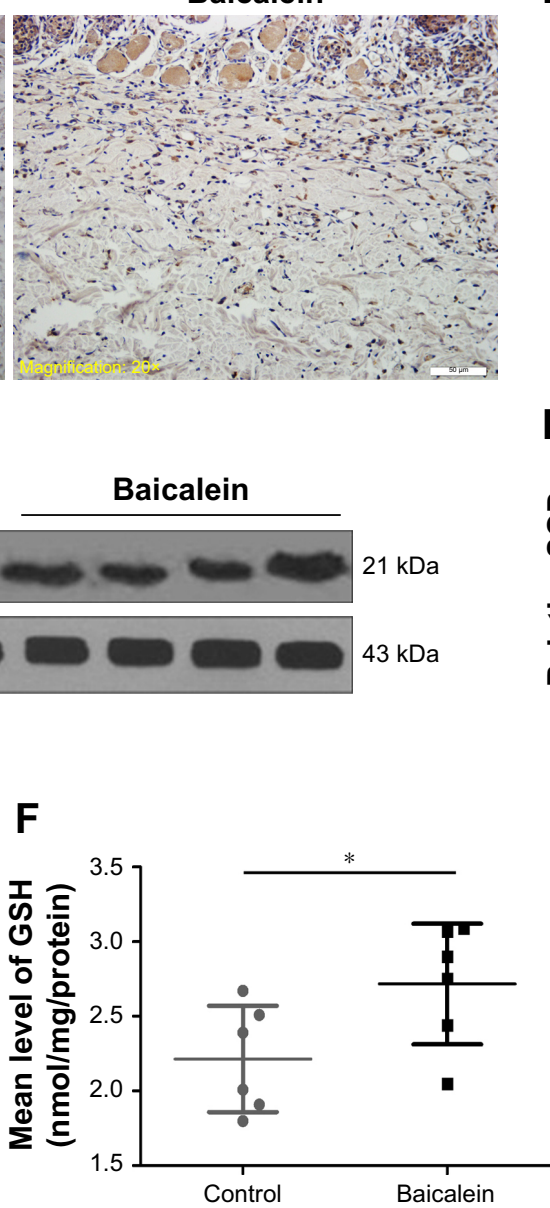

B

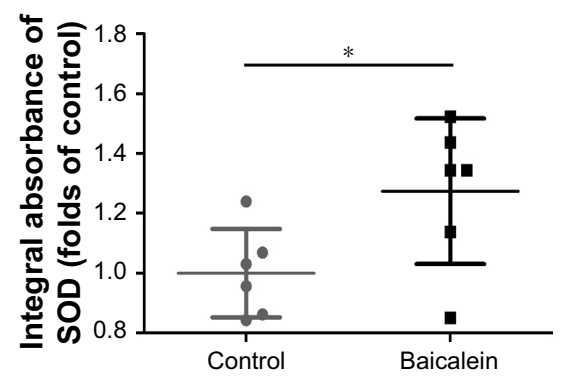

D

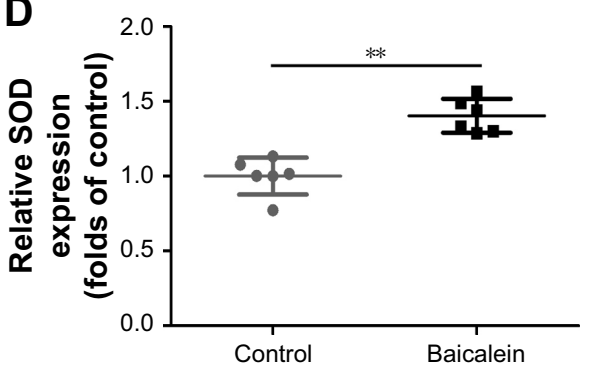

G

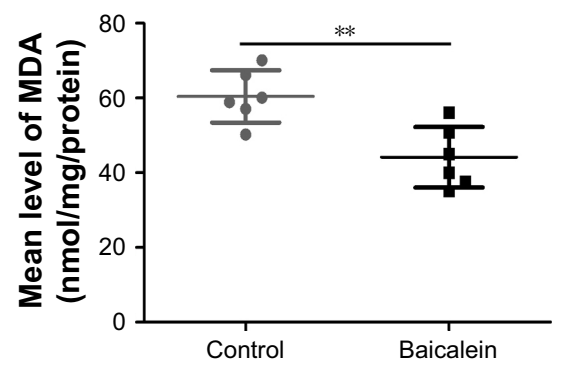

Figure 5 Baicalein reduced oxidative stress in ischemic flap regions.

Notes: (A) Immunohistochemical evaluation of SOD expression in ischemic flaps (original magnification, $\times 200$; scale bar, $50 \mu \mathrm{m}$ ). (B) ODs indicative of SOD expression. (C) Western blotting for SOD on Day 7. All gels were run under the same experimental conditions, and cropped images of the blots are presented. (D) ODs indicative of SOD expression are shown. (E-G) The levels of SOD, GSH, and MDA in ischemic flaps were measured using the xanthine oxidase, modified dithiobisnitrobenzoic acid, and modified thiobarbituric acid methods, respectively. Values are mean $\pm S D ; n=6$ per group. Significant differences are indicated by $* P<0.05, * * P<0.01$.

Abbreviations: GSH, glutathione; MDA, malondialdehyde; SOD, superoxide dismutase.

apoptosis, and leukocyte/endothelial interactions. ${ }^{25}$ Apoptosis and oxidative stress induced by free radicals are the principal contributors to necrosis. ${ }^{26}$ Baicalein reduced apoptosis by modulating the PI3K/Akt signaling pathway. ${ }^{13}$ Baicalein ameliorated tubulointerstitial fibrosis by inducing myofibroblastic apoptosis via the intrinsic mitochondrial pathway. ${ }^{27,28}$ Baicalein increased SOD and GSH peroxidase activities and reduced the MDA level, alleviating oxidative stress and affording cytoprotection via modulation of the activities of extracellular regulated protein kinases and the HO-1 signaling pathway. ${ }^{29,30}$

We evaluated the effects of baicalein on oxidative stress and apoptosis during ischemia of random skin flaps. SOD, GSH, and MDA are three important markers of oxidation. SOD, an antioxidant enzyme, removes oxygen free radicals. ${ }^{31}$ GSH, a major intracellular antioxidant, also removes free radicals and facilitates superoxide anion dismutation. ${ }^{17}$
MDA is a secondary product of lipid peroxidation, and the MDA level reflects the extent of oxidation-induced tissue injury. ${ }^{32}$ Therefore, we measured SOD, GSH, and MDA levels as markers of oxidative stress status. Baicalein significantly increased SOD and GSH levels and reduced that of MDA. Baicalein reduced oxidative stress. Caspases, a family of cysteine proteases, play critical roles in apoptosis; CASP3 is activated by a series of cleavage events to generate cleaved CASP3, a key indicator of apoptosis. ${ }^{33,34}$ We measured the cleaved CASP3 level to monitor apoptosis, and the level was significantly lower in the baicalein group than in the control group. Thus, baicalein reduced apoptosis.

Our work could be strengthened by addition of serum baicalein concentrations at various times after injection, to explore when the steady state is attained and to confirm physiologically relevant levels. Moreover, the therapeutic effects 
should be compared with those of growth factors, glucocorticoids, and oxygen free radical scavengers in future studies.

\section{Conclusion}

Baicalein stimulated angiogenesis and reduced oxidative stress and apoptosis, improving random skin flap viability. Further experimental and clinical studies are required.

\section{Acknowledgments}

This study was supported by the Natural Science Foundation of China (grant no 81601705 to KZ and 81572227 to HX), the Zhejiang Provincial Medicine and Health Technology Project (grant no 2017 KY472 to KZ), the Science Technology Department of Zhejiang Province Project (grant no 2017C33024 to DL), and the Wenzhou Science and Technology Bureau Foundation (grant no 2016Y0350 to JD, 2015 Y0060 to SL, and Y20160039 to DL).

\section{Authors contributions}

RL and JL wrote the first draft. Shihen Li, JD, and HW prepared the figures and collected the samples. GX, Shi Li, $\mathrm{YH}$, and DL analyzed the data. $\mathrm{KZ}$ designed the experiment. WG, JK, HX, and KZ revised the manuscript. All authors contributed toward data analysis, drafting and revising the paper and agree to be accountable for all aspects of the work. All authors reviewed and approved the final manuscript.

\section{Disclosure}

The authors report no conflicts of interest in this work.

\section{References}

1. Lee MS, Ahmad T, Lee J, et al. Dual delivery of growth factors with coacervate-coated poly(lactic-co-glycolic acid) nanofiber improves neovascularization ina mouse skin flap model. Biomaterials. 2017;124:65-77.

2. Deheng C, Kailiang Z, Weidong W, et al. Salidroside promotes random skin flap survival in rats by enhancing angiogenesis and inhibiting apoptosis. J Reconstr Microsurg. 2016;32(8):580-586.

3. Douglas B, Buchholz RR. The blood circulation in pedicle flaps: an accurate test for determining ITS efficiency. Ann Surg. 1943;117(5): 692-709.

4. Roh TS, Jung BK, Yun I, Lew DH, Kim YS. Effect of botulinum toxin A on vasoconstriction and sympathetic neurotransmitters in a murine random pattern skin flap model. Wound Repair Regen. 2017;25(1):75-85.

5. Lin R, Chen H, Callow D, et al. Multifaceted effects of astragaloside IV on promotion of random pattern skin flap survival in rats. Am J Transl Res. 2017;9(9):4161-4172.

6. Cheng L, Chen T, Li H, et al. Effects of Tirofiban on Random Skin Flap Survival in Rats. J Reconstr Microsurg. 2018;34(2):138-144.

7. Sheng L, Yang M, Li H, et al. Transplantation of adipose stromal cells promotes neovascularization of random skin flaps. Tohoku J Exp Med. 2011;224(3):229-234.

8. Cheng CS, Chen J, Tan HY, et al. Scutellaria baicalensis and Cancer Treatment: Recent Progress and Perspectives in Biomedical and Clinical Studies. Am J Chin Med. 2018;46(1):25-54.
9. Chan EWC, Wong SK, Chan HT. Casticin from Vitex species: a short review on its anticancer and anti-inflammatory properties. J Integr Med. 2018;16(3):147-152.

10. Zhu XX, Yang L, Li YJ, Yj L, et al. Effects of sesquiterpene, flavonoid and coumarin types of compounds from Artemisia annua L. on production of mediators of angiogenesis. Pharmacol Rep. 2013;65(2): 410-420.

11. Lee SI, Kim SY, Park KR, Kim EC. Baicalein Promotes Angiogenesis and Odontoblastic Differentiation via the BMP and Wnt Pathways in Human Dental Pulp Cells. Am J Chin Med. 2016;44(7):1457-1472.

12. Wei N, Wei Y, Li B, Pang L. Baicalein promotes neuronal and behavioral recovery after intracerebral hemorrhage via suppressing apoptosis, oxidative stress and neuroinflammation. Neurochem Res. 2017;42(5):1345-1353.

13. Liu C, Wu J, Xu K, et al. Neuroprotection by baicalein in ischemic brain injury involves PTEN/AKT pathway. J Neurochem. 2010;112(6): $1500-1512$.

14. Kumar M, Kasala ER, Bodduluru LN, Dahiya V, Lahkar M. Baicalein protects isoproterenol induced myocardial ischemic injury in male Wistar rats by mitigating oxidative stress and inflammation. Inflamm Res. 2016;65(8):613-622.

15. Liang W, Huang X, Chen W. The effects of baicalin and baicalein on cerebral ischemia: a review. Aging Dis. 2017;8(6):850-867.

16. Zhou KL, Zhang YH, Lin DS, Tao XY, Xu HZ, Hz X. Effects of calcitriol on random skin flap survival in rats. Sci Rep. 2016;6:18945.

17. Chen L, Zhou K, Chen H, et al. Calcitriol promotes survival of experimental random pattern flap via activation of autophagy. Am J Transl Res. 2017;9(8):3642-3653.

18. Liu A, Huang L, Fan H, et al. Baicalein pretreatment protects against liver ischemia/reperfusion injury via inhibition of NF- $\kappa B$ pathway in mice. Int Immunopharmacol. 2015;24(1):72-79.

19. Chao HM, Chuang MJ, Liu JH, et al. Baicalein protects against retinal ischemia by antioxidation, antiapoptosis, downregulation of HIF- $1 \alpha$, VEGF, and MMP-9 and upregulation of HO-1. JOcul Pharmacol Ther. 2013;29(6):539-549.

20. van Leyen K, Kim HY, Lee SR, et al. Baicalein and 12/15-lipoxygenase in the ischemic brain. Stroke. 2006;37(12):3014-3018.

21. Saul D, Kling JH, Kosinsky RL, et al. Effect of the lipoxygenase inhibitor baicalein on muscles in ovariectomized rats. J Nutr Metab. 2016;2016:3703216.

22. Miocinovic R, Mccabe NP, Keck RW, et al. In vivo and in vitro effect of baicalein on human prostate cancer cells. Int J Oncol. 2005;26(1): 241-246.

23. Harder Y, Amon M, Erni D, Menger MD. Evolution of ischemic tissue injury in a random pattern flap: a new mouse model using intravital microscopy. J Surg Res. 2004;121(2):197-205.

24. Aydogan H, Gurlek A, Parlakpinar H, et al. Beneficial effects of caffeic acid phenethyl ester (CAPE) on the ischaemia-reperfusion injury in rat skin flaps. J Plast Reconstr Aesthet Surg. 2007;60(5):563-568.

25. Massberg S, Messmer K. The nature of ischemia/reperfusion injury. Transplant Proc. 1998;30(8):4217-4223.

26. Song K, Zhang M, Hu J, et al. Methane-rich saline attenuates ischemia/ reperfusion injury of abdominal skin flaps in rats via regulating apoptosis level. BMC Surg. 2015;15:92.

27. Wang W, Zhou PH, Xu CG, Cg X, et al. Baicalein ameliorates renal interstitial fibrosis by inducing myofibroblast apoptosis invivo and invitro. BJU Int. 2016;118(1):145-152.

28. Xia J, He LQ, Su X. Interventional mechanisms of herbs or herbal extracts on renal interstitial fibrosis. J Integr Med. 2016;14(3):165-173.

29. Liu C, Wu J, Gu J, et al. Baicalein improves cognitive deficits induced by chronic cerebral hypoperfusion in rats. Pharmacol Biochem Behav. 2007;86(3):423-430.

30. Chen YC, Chow JM, Lin CW, Wu CY, Shen SC. Baicalein inhibition of oxidative-stress-induced apoptosis via modulation of ERKs activation and induction of HO-1 gene expression in rat glioma cells C6. Toxicol Appl Pharmacol. 2006;216(2):263-273. 
31. Scandalios JG. Oxygen Stress and Superoxide Dismutases. Plant Physiol. 1993;101(1):7-12.

32. Esterbauer H. Cytotoxicity and genotoxicity of lipid-oxidation products. Am J Clin Nutr. 1993;57(5 Suppl):779S-786S.

33. Larsen BD, Rampalli S, Burns LE, et al. Caspase 3/caspase-activated DNase promote cell differentiation by inducing DNA strand breaks. Proc Natl Acad Sci U S A. 2010;107(9):4230-4235.
34. Slee EA, Adrain C, Martin SJ. Executioner caspase-3, -6, and -7 perform distinct, non-redundant roles during the demolition phase of apoptosis. J Biol Chem. 2001;276(10):7320-7326.

\section{Publish your work in this journal}

Drug Design, Development and Therapy is an international, peerreviewed open-access journal that spans the spectrum of drug design and development through to clinical applications. Clinical outcomes, patient safety, and programs for the development and effective, safe, and sustained use of medicines are the features of the journal, which has also been accepted for indexing on PubMed Central. The manuscript management system is completely online and includes a very quick and fair peer-review system, which is all easy to use. Visit http://www.dovepress.com/testimonials.php to read real quotes from published authors.

Submit your manuscript here: http://www.dovepress.com/drug-design-development-and-therapy-journal 